\title{
Seismic Event Interpretation Using Fuzzy Logic and Neural Networks
}

\author{
W. J. Maurer \\ F. U. Dowla
}

January, 1994






\section{DISCLAIMER}

This document was prepared as an acccount of work sponsored by an agency of the United States Government. Neither the United States Govermment nor the University of California nor any of their employees, makes any warranty, express or implied, or assumes any legal liability or responsibility for the accuracy, completeness, or usefulness of any information, apparatus, product, or process disclosed, or represents that its use would not infringe privately own rights. Reference herein to any specific commercial products, process, or service by trade name, trademark, manufacturer, or otherwise, does not necessarily constitute or imply its endorsement, recommendation, or favoring by the United States Government or the University of California. The views and opinions of authors expressed herein do not necessarily state or reflect those of the United States Government or the University of California, and shall not be used for advertising or product endorsement purposes.

This report has been reproduced directly from the best available copy.

Available to DOE and DOE contractors from the Office of Scientific and Technical Information P.O. Box 62, Oak Ridge, TN 37831

Prices available from (615) 576-8401, FTS 626-8401

Available to the public from the National Technical Information Service

U.S. Department of Commerce 5285 Port Royal Rd., Springfield, VA 22161 


\title{
Seismic Event Interpretation Using Fuzzy Iıogic and Neural Networks
}

\author{
William J. Maurer and Farid U. Dowla \\ Treaty Verification Program \\ Lawrence Livermore National Laboratory
}

\begin{abstract}
In the computer interpretation of seismic data, unknown sources of seismic events must be represented and reasoned about using measurements from the recorded signal. In this report, we develop the use of fuzzy logic to improve our ability to interpret weak seismic events. Processing strategies for the use of fuzzy set theory to represent vagueness and uncertainty, a phenomena common in seismic data analysis, are developed. A fuzzy-assumption based truth-maintenance-inferencing engine is also developed. Preliminary results in interpreting seismic events using the fuzzy neural network knowledge-based system are presented.
\end{abstract}

\section{Introduction}

In recent years, much effort has gone into studying the problem of computer interpretation of seismic events. Seismic monitoring networks deployed worldwide for the verification of nuclear test ban treaties and nonproliferation will annually detect hundreds of thousands of seismic events (of magnitudes greater than 2) both natural and cultural, that will have to be interpreted. An automated seismic data interpretation system is essential for this task.

Over the past decade, a number of experimental automated or semi-automated systems (Johnson et al., 1987; Bache et al., 1990) have been developed. We now understand the benefits and limitations of these systems more clearly. However, most interpretation systems developed to date have relied on seismic arrays. Seismic arrays typically consists of 20 to 30 stations; these arrays are powerful but expensive receivers. The focus of this work is on using simple one-or three-component stations 
for high-level seismic interpretation. In the context of a worldwide nonproliferation environment, we believe that simple stations, which are significantly less expensive, might be widely deployed.

A simple three-component station, however, allows fewer measurements that can be extracted from the data. For example, a single three-component station, unlike a seismic array, does not allow measurement of the $\mathrm{Lg}$ phase velocity, an important measurement in automated analysis. Without the Lg phase velocity measurement, two of the critical tasks phase identification and phase association become much more difficult. (In seismic interpretation phase identification is the process of identifying the phase types: $\mathrm{Pn}, \mathrm{Pg}, \mathrm{Lg}$, Rayleigh, Love; phase association refers to the grouping of these phases by seismic events.)

From our experience, interpretation of seismic events with data from a single three-component station and with a relatively small set of logical if-then rules, results, too frequently, in false interpretations of the seismic phase identification. and association. The reason for this is partly because different types of sources (such as teleseismic, regional, and local earthquakes, mining explosions, and other cultural events) generate waveforms that are not only very different from one class to another, but are also nonuniform within a single class of events. The class of seismic events include: regional events, teleseismic events, local events, and other events due to natural phenomena and cultural activities. With limited measurements, it is difficult to develop a set of "rules" that can handle the variation in the data from the different types of events.

In this paper, we propose an approach to seismic interpretation systems. In this approach, we show that a Self Organizing Neural Network (SONN) can be used as a front-end to a knowledge-based interpretation system (Maurer et al., 1992) to address vagueness and ambiguities and to improve event interpretation. Vagueness and ambiguity are inherent in interpreting seismic data from a simple station; the proposed scheme uses the SONN as a fuzzy associative memory (Kosko, 1992) to a fuzzy inferencing engine to deal with these uncertainties.

\section{Seismic Interpretation: Structure and Approach}

The specific problem addressed here can be summarized as follows. Given a simple seismic (3-, 2-, or 1-component) station, what is the best general strategy for interpreting detected seismic events, despite the variation in the characteristics of 
the observed events both within each class and among the various classes of seismic events. From the results of this study, we draw two main conclusions. First, in seismic event interpretation, multiple fuzzy interpretations prior to a precise interpretation of an event are almost always useful. Second, multiple fuzzy interpretations may often be the critical first step in successful event interpretation.

For this approach, we assume that event detection and data segmentation have been performed. Event detection is the task of determining the onset of arrival in the data due to a seismic event such as an earthquake or an underground explosion. Data segmentation is the task of delimiting the segment of the data where phase arrivals due to an event can be measured. Before phase identification and phase association are completed, the seismic event is fuzzy classified according to a broad category of candidate classes, such as teleseismic, regional, local, mining, vehicular, or other cultural sources. Following such a classification, a fuzzy rulebased system can be employed to perform the rest of the interpretation for each possible class. Once a number of fuzzy interpretations are available, we can make a precise interpretation by analyzing the set of interpretations as a whole. The fuzzy-classification-first approach is shown in Figure 1. We prefer to break the complex task of seismic event interpretation into various subtasks and then attack each problem with a methodology that is best suited for that particular subtask. This allows us to take advantage of methods from various fields, such as signal processing, expert systems, neural networks, and fuzzy set theory.

\section{$3 \quad$ Fuzzy Set Theory}

"Fuzzy set theory" is used to refer to a class of methods that propose a logical treatment of imperfect knowledge. Imperfect knowledge can be expressed in terms of vagueness or uncertainty. Issues of vagueness and uncertainty have become important with the emergence of advanced information systems equipped with automated reasoning capabilities. The underlying problem addressed by fuzzy set theory is how to represent a statement in such a way that its meaning can span the spectrum of vagueness, from ambiguous to precise. Likewise, truth can span the spectrum of uncertainty, from unknown to completely known. Since fuzzy logic systems rely on these concepts and employ these concepts through linguistics variables, we expand on these in the following subsections. 


\subsection{Vagueness}

Vagueness is associated with the difficulty of making sharp or precise distinctions in the world. For example, in seismic analysis, a weak arrival creates a detection that is vague - it cannot be delimited by clear yes or no decision. Fuzzy set theory spans the spectrum of vagueness by using a membership function in some domain. Membership values (in fuzzy sets) are indicated by a value on the range $[0.0,1.0]$, with 0.0 representing absolute non-membership, and 1.0 representing complete membership. For example, let us take the statement:

"There is a phase arrival at time $T$ "

If the short-time over long-time signal amplitude (STA/LTA) level is very high (for example, a STA/LTA value greater than 5), we might assign to this statement a truth value of 0.80 . The statement could be translated into set theory terminology as follows:

"Time $\mathrm{T}$ is a member of the set of arrivals."

The above statement would be rendered symbolically with fuzzy membership function:

$\operatorname{mARRIVAL}(\mathrm{T})=0.80$

where the $m$ in mARRIVAL is the membership function, operating in this case on the fuzzy set of arrivals, which returns a value between 0.0 and 1.0.

It is important to point out the distinction between fuzzy systems and probability. Both operate over the same numeric range, and at first glance both have similar values: 0.0 representing false (or non- membership), and 1.0 representing true, (or membership). However, there is a distinction between the two statements: The probabilistic approach yields the natural-language statement, "There is an $80 \%$ chance that there is an arrival at time $T$, , while the fuzzy terminology corresponds to "Time T's degree of membership within the set of arrivals is 0.80 ." The semantic difference is significant. The first view supposes that time $\mathrm{T}$ does or does not correspond to an arrival; it is just that we only have an $80 \%$ chance of knowing which set $\mathrm{T}$ belongs to. By contrast, fuzzy terminology supposes that "time $\mathrm{T}$ is more or less" an arrival corresponding to the value of 0.80 . In seismic analysis, it is natural to work with fuzzy membership functions. 


\subsection{Truth}

Truth is generally understood as the conformity of a statement and the actual state of facts to which it refers. Within an information system, a degree of truth is a measure of agreement between the representation of the meaning of a statement and the representation of what is actually known about reality. Zadeh (1982) proposed the meaning of a statement to be constraints restricting the values of variables that are implicit in the statement. What is known of reality is supposedly stored in a database in the form of statements. The truth of a statement is derived from a matching procedure between the meaning of tire statement (variable constraints) and the contents of the database. Depending on the precision of the query and the database, the query can be asserted, refuted, or only partially known (full of uncertainty).

\subsection{Linguistic Variables}

The concept of a linguistic variable provides a means of representing the meaning of a statement. A linguistic variable is a variable whose values are words or sentences in a language (Zadeh, 1975). For example, ARRIVAL is a linguistic variable if its values are linguistic rather than numerical (e.g., strong, not weak, weak, not very weak, very weak rather than STA/LTA values of $5,4,3,2,1)$. In specific terms, a linguistic variable is defined by a quintuple $(H, T(H), U, G, M)$ where $H$ is the name of the variable, $\mathrm{T}$ is the term-set of $\mathrm{H}$, which is its collection of linguistic values, $U$ is the universe of discourse (i.e. the domain being modeled), $G$ is a syntactic grammar that generates the terms in $T(H)$, and $M$ is a semantic rule that associates its meaning with each linguistic value $X, M(X)$. The meaning of a linguistic value $X$ is characterized by a compatibility function , $\mathrm{c}: \mathrm{U} \mapsto[0,1]$, which associates with each $\mathrm{u}$ in $\mathrm{U}$ its compatibility with $\mathrm{X}$. Thus, the compatibility of STA/LTA greater than 5 with strong might be 0.8 , whereas that of 4 might be 0.5 . The function of the semantic rule is to relate the compatibilities of the primary terms of a composite linguistic value (e.g., strong and weak) in not very strong and not very weak to the compatibility of the composite values. The meaning of the primary terms is context dependent and is specified a priori. In addition to the primary terms, a composite linguistic value may contain hedges (e.g., very, quite, extremely), connectives (e.g., and, or), or negation, which are treated as context independent nonlinear operators that modify the meaning of their operators in a specified fashion. Truth can 
be treated as a linguistic variable with linguistic values such as true, very true, completely true, not very true, and untrue, leading to what is called fuzzy logic.

\section{$4 \quad$ Fuzzy Truth Maintenance System}

Our motivation for using a truth maintenance system is the way in which seismologists hypothesize interpretations based on a set of beliefs. If the belief in an interpretation leads to a contradiction, then the interpretation is marked as unbelievable. For example, it is known that, in a local regional network, a teleseismic event should be interpreted as teleseismic at all the stations. If it is not classified as teleseismic at all the stations, then the inferencing chain along a teleseismic belief must be incorrect, and the partial interpretation is disbelieved. We developed a system, FUZZSEA, that employs a Truth Maintenance System (TMS) (Doyle, 1979) to handle belief revision of facts. Furthermore, it is an Assumption-based Truth Maintenance System (ATMS) (deKleer, 1984). ATMSs are typically best in situations where there are a number of solutions and all of them must be found. The existence of multiple interpretations is an indicator that there are not enough rules to reject invalid interpretations and of the inherent fuzziness in the problem.

\section{Knowledge Representation Scheme}

We use a semantic network formalism as the knowledge representation scheme. Facts are represented as a <node, link, node> triple (Johnson et al., 1987). A network is specified by the set of facts it represents. Figure 2 shows the sr mantic network formalism. The example network in Figure 2 represents two seismic monitoring stations that have each detected an event which is represented by a seismic waveform. At one station the event has been classified as a teleseismic event, and as a local event at the other station. The teleseismic waveform contains a phase arrival pick that has been labeled as a $\mathrm{Pn}$ phase. The $\mathrm{Pn}$ phase is located $\mathbf{1 2 1 . 5}$ seconds after the beginning of the waveform. The local waveform contains a phase arrival pick that has been labeled as a $\mathrm{Pg}$ phase. The $\mathrm{Pg}$ phase is located 43.8 seconds after the beginning of the waveform. The <node. Iink, node> triples used to represent these facts are shown below:

<station instance node348>

<station instance node351> 
<node348 waveform node349>

〈node349 class 'teleseismic>

<node349 pick node350>

<nòde350 phase 'Pn>

<node350 begin-time 121.5> <node351 waveform node352>

<node352 class 'local>

<node352 pick node353>

<node353 phase ' $P$ g >

<node353 begin-time 43.8> ...

Access to the net is achieved by a pattern-matching process. Patterns contain variables that are bound during the matching process. A pattern usually matches more than one subnet. Each match yields a different set of variable bindings. As an example, consider the pattern below designed to match the facts just described above:

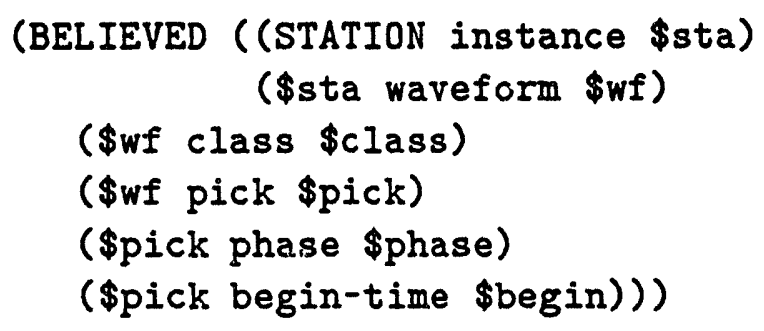

Applied to the net in Figure 2, it yields two variable binding sets:

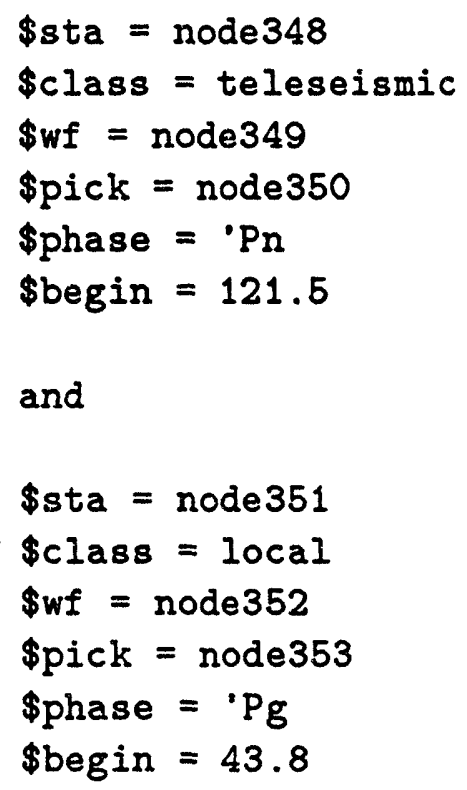


The knowledge base consists of a set of rules of the form (rule-name pattern action). Each set of variable bindings for the pattern gives rise to a separate instantiation of the rule. Variable bindings in the rule instantiation can be used in the rule action. For example, the rule:

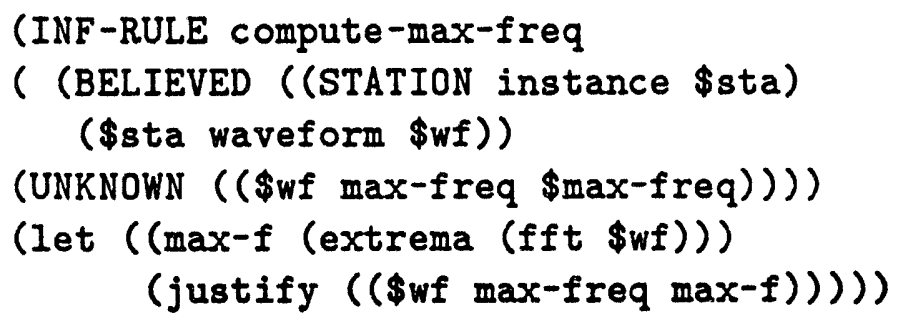

evaluates an FFT for a feature, extracts the extrema, and adds an appropriate subnet to represent the computed value. There are two instantiations for the rule in the net shown in Figure 2.

The ATMS is based on the concept of assumption sets being labeled as either IN or OUT (deKleer, 1984). We briefly describe how assumptions sets are modeled in FUZZSEA below for the interested reader.

An assumption set is a subset of the known facts. The set of all possible assumption sets is the power set of the known facts. If an assumption set is labeled as IN, this means the facts are consistent with one another; they can all be true at the same time. OUT means the facts are inconsistent; they cannot all be true at the same time. If an assumption set $A$ is a subset of an assumption set $B$, then if A is OUT, this implies that B is OUT. Each fact has an assumption set associated with it. When a rule fires there are a set of antecedent facts, A1...An, and a set of created facts, $\mathrm{C} 1$...Cn. When a $\mathrm{Ci}$ is created, the assumption set for the created fact depends on whether the fact is created as a GIVEN fact, a JUSTIFY fact, or an ASSUME fact.

The set of assumptions for a GIVEN fact is just the fact you are creating because it is a given truth. $\mathrm{AS}(\mathrm{Ci})=\mathrm{Ci}$.

On the other hand, the assumption set for a JUSTIFY fact is the union of the assumption sets of the antecedent facts for the rule because you are declaring the created fact as true based on the consistency of the antecedents collective truth: $\mathrm{AS}(\mathrm{Ci})=$ union $(\mathrm{AS}(\mathrm{A} 1 . . \mathrm{An}))$. 
Finally, the assumption set for an ASSUME fact is the union of the assumption sets of the antecedent facts for the rule plus the fact you just created because you are supposing or hypothesising the created fact as true based on the consistency of the antecedents collective truth: $\mathrm{AS}(\mathrm{Ci})=$ union( $\mathrm{AS}(\mathrm{A1}$..An $))+\mathrm{Ci}$.

Non-monotonic reasoning is achieved by using the CONTRADICT <pattern> function. This causes the ATMS to form the union the assumption sets of all the facts in <pattern>, then search through the assumption database and mark all supersets of the resulting assumption set as OUT.

\section{Fuzzy System Architecture}

The goal of the design of the fuzzy inference engine is to be able to incorporate vagueness and uncertainty into a high level reasoning system in the context of a particular application. This motivation is based on the observation that a context independent definition of what fuzziness really means does not exist. To accomplish this context independence, we identified what is common to all applications that deal with fuzzy information and inferencing: fuzzy measures are required to compute the degree of membership of elements of a fuzzy set; meaning representation is necessary to provide a mechanism by which to determine the meaning of a statement; rules of inference are necessary to infer new information in a data base. In this work we used the Common Lisp Object System (CLOS) to extend the ATMS logical inference engine described above to incorporate fuzzy set theory by creating a linguistic variable object class hierarchy. During pattern matching, if the link in a <node. Iink, node> triple used to represent a fact is a linguistic variable, we compute the compatability function of the fact being matched. This compatability value gets propagated onto instantiations further down along the pattern by means of the user-defined CONJUCTION-FUNCTION which takes a list of compatability values as arguments and returns the conjunction of those values. When a pattern is completed and a rule is fired, the compatability value of that pattern is propagated to the facts created by the rule action.

A user-defined value called the CROSSOVER-POINT is set to a number between 0.0 and 1.0. The CROSSOVER-POINT represents the transition from false to true and is used in pattern matching as follows:

BELIEVED facts are compatability value >= CROSSOVER-POINT

DISBELIEVED facts are compatability value < CROSSOVER-FOINT 
KNOWN facts are compatability value $>=0$

UNKNOWN facts are nil

When a fact is created with GIVEN, JUSTIFY, or ASSUME, the assumption set for the fact is computed as done in the ATMS logical system. The assumption set represents the union of facts upon which this fact depends. Since a union operation is used to construct these sets, we have a user-defined UNION-ASSUMPTIONFUNCTION, which takes a list of compatability values and returns the union of those values. To express the creation of a fuzzy fact, the fact link must be a linguistic variable object. If the value of the linguistic variable is a fuzzy expression of its terms, then the expression must be defuzzified. Defuzzification is the process of mapping the compatability value of a linguistic variable into a symbolic representation using the terms of the linguistic variable along with hedges and connectives.

When a CONTRADICT is executed all the assumption sets that are supersets of the contradicted fact are marked as OUT in the ATMS logical system. In the fuzzy system, the interpretation is that we are contradicting a fuzzy restriction, this is equivalent to using the NOT modifier on a fuzzy restriction. If we view the assumption set as a fuzzy restriction, then we can apply the NOT to the assumption set; (e.g., NOT(XvXvX) = 1 - assumption set compatability value).

\section{$7 \quad$ Fuzzy Logic and Neural Networks}

A neural network maps points in some input space to points in some output space (Maurer et al., 1992). A fuzzy associative memory maps fuzzy sets in some input space to fuzzy sets in some output space, which may overlap (Kosko, 1992; Dubois, 1988). In this study, we use a Self Organizing Neural Network (SONN), as a Fuzzy Associative Memory (FAM). The training set for the SONN used 43 events. The number of events in each class as labeled by the seismic analyst are: automobiles (9), noise (6), local seismic (9), regional seismic (10), and teleseismic(9). Table 1 shows how these analyst-classified events are distributed throughout the seven clusters formed by the Kohonen model. We used various numbers of clusters and found that the cluster grouping results were best when we used seven clusters.

Table 1: Fuzzy Self-Organizing Neural Network Clusters 


\begin{tabular}{|c|c|c|c|c|c|}
\hline SONN's Cluster & \multicolumn{5}{|c|}{ Analyst's Cluster } \\
\hline Description & Auto & Noise & Local & Regional & Teleseismic \\
\hline K-1: broadband, short-duration & $0(.0)$ & $1(.125)$ & $5(.625)$ & $2(.25)$ & $0(.0)$ \\
\hline K-2: broadband, periodic & $4(1)$. & $0(.0)$ & $0_{(.0)}$ & $0_{(.0)}$ & $0(.0)$ \\
\hline K-3: noisy & $1(.143)$ & $4(.571)$ & $2(.246)$ & $0(.0)$ & $0(.0)$ \\
\hline K-4: low SNR & $O_{(.0)}$ & $0_{(.0)}$ & 1 (.s33) & 1 (.ss3) & 1 (.sss) \\
\hline K-5: broadband, decay & $0(.0)$ & $0(.0)$ & $0(.0)$ & $2(.286)$ & $5(.714)$ \\
\hline K-6: midband, decay & $0(.0)$ & $0(.0)$ & $0_{(.0)}$ & $5(.714)$ & $2(.286)$ \\
\hline K-7: periodic, multiple & $4(.571)$ & $1(.143)$ & $1(.143)$ & $0(.0)$ & $1(.143)$ \\
\hline
\end{tabular}

The clusters are labeled $\mathrm{K}-1$ through $\mathrm{K}-7$, and a qualitative description of the spectrograms of each cluster is given in the first column of the Table 1 . In this table, the cluster groups formed by the Kohonen nodes are grouped according to row, whereas the analyst-classification is shown in the columns. The columns represent the distzibution of the SONN nodes over a type of event, and the rows represent the distribution of the types of events at a SONN node. Note that there are two numbers in each entry; the larger script integer represents the number of events, and the smaller script real number is the same number normalized by the number of events in that cluster (or row). We will refer to this number (between 0 . and 1.) by the term event probability. For example, the event probability, 0.571 of cluster $K-\rho$ and class noise is a measure of the probability that an event is noise if it were to be categorized in cluster $K-3$.

The integration of the SONN with FUZZSEA requires that the SONN be on the order of a few seconds to run in a real monitoring environment, and that it be able to recognize novel and unexpected events. The second requirement is met by modifying the Kohonen network so that new classes are generated with novel events. After learning has been successfully completed with a number of training events, the network weights are fixed, and the network, used in the manner as described below, is fast enough to meet the speed requirement.

To recognize a known pattern or novel event, the Kohonen network first matches the input pattern with the winning cluster by computing the correlation coefficient between the two. When the correlation is below a certain threshold (0.9), then the pattern is classified as unknown. Otherwise the pattern is in each cluster (K1-Kr) with a different degree of match. This results in activating the event probability appropriately. These fuzzy classifications are inserted into the knowledge base as facts with an initial compatability value to start the inferencing along a hypothesis. The approach we take is to develop sets of rules specific to each of the different 
classes of events, and truth maintenance rules that recognize contradictions between the classes. Each initial hypothesis is explored by the inference engine, and a set of fuzzy interpretations is obtained.

\subsection{Seismic Classification Linguistic Variable}

Below is the SEISMIC-CLASSIFICATION linguistic variable used to model the seismic classification fuzzy set found in the weights of the Kohonnen neural network.

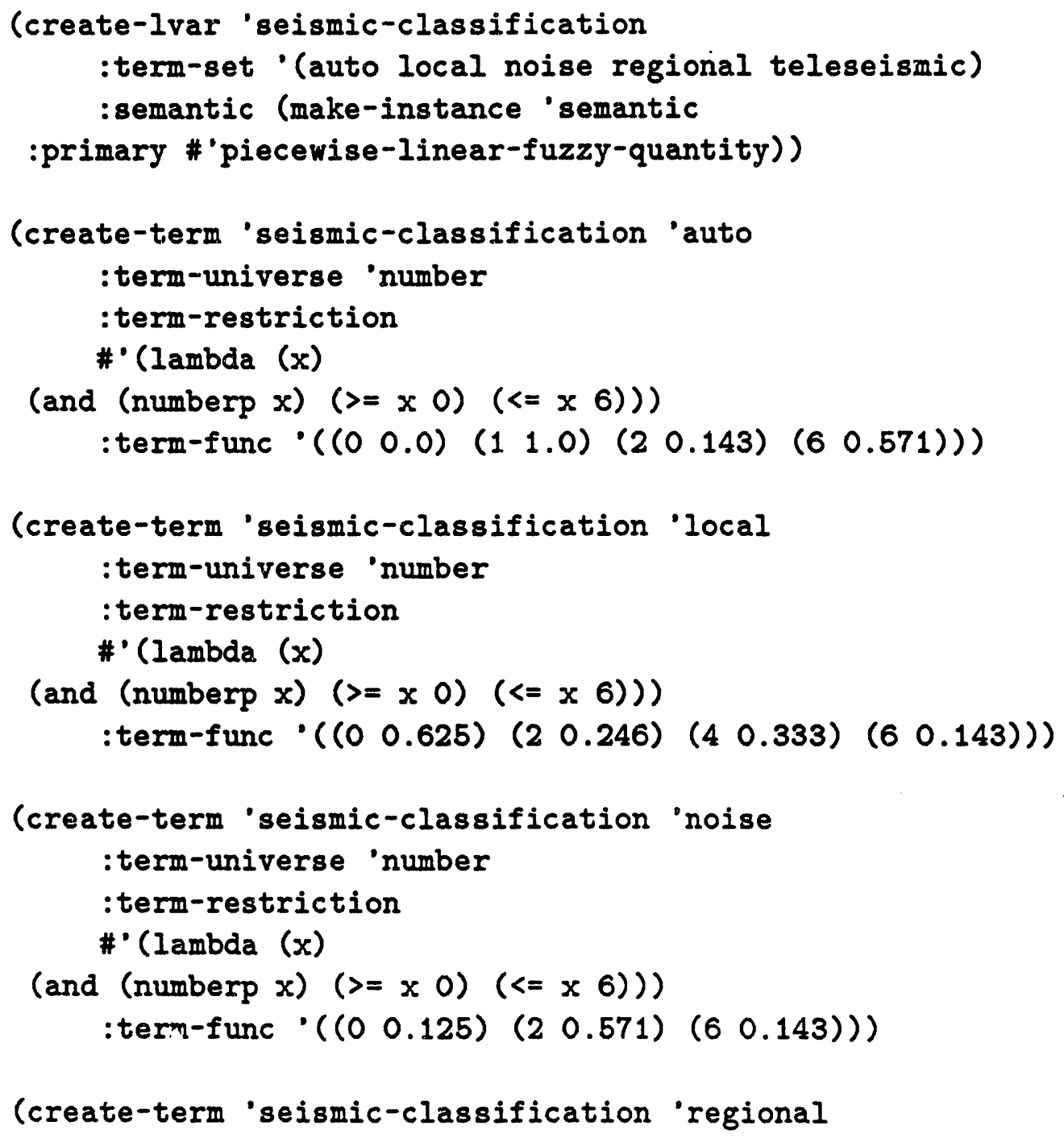






The function CREATE-LVAR creates a linguistic variable object seismic-classification. seismic-classification can take on the different linguistic values from the term set: auto local noise regional teleseismic. The piecewise-linear-fuzzy-quantity function is used to compute the compatibility function, of the terms. Each of the linguistic values in the term-set is created with the function CREATE-TERM. CREATE-TERM creates a linguistic value object and defines the compatibility function which is passed as an argument to the linguistic variable object semantic function piecewiselinear-fuzzy-quantity.

In this example, the compatibility functions represent the distributions of the various classes throughout the clusters. Since the weights in the clusters are trained to represent the mean examplar for that cluster, we can compute the correlation coefficient between weights in each cluster to determine the distribution. Referring to Table 1 , we can see cluster $\mathrm{K} 2$ is most representative of an automobile If we compute the correlation of the $\mathrm{K} 2$ weights with each clusters' weights, we see the following distribution:
2 wrt $0=0.926701$
2 wrt $1=1.000000$
2 wrt $2=0.884439$
2 wrt $3=0.899197$
2 wrt $4=0.890370$
2 wrt $5=0.884320$
2 wrt $6=0.910236$ 
where ( $\mathrm{x}$ wrt $\mathrm{y}$ ) means event $x$ with respect to class $y$. With a large number of events, these distributions can be used to compute a smooth function, thus allowing us to to describe membership of a given event to the different classes more precisely.

\subsection{Example: A Truth Maintenance Rule}

Below is an example of a TMS rule to filter out an invalid "teleseismic" hypothesis:

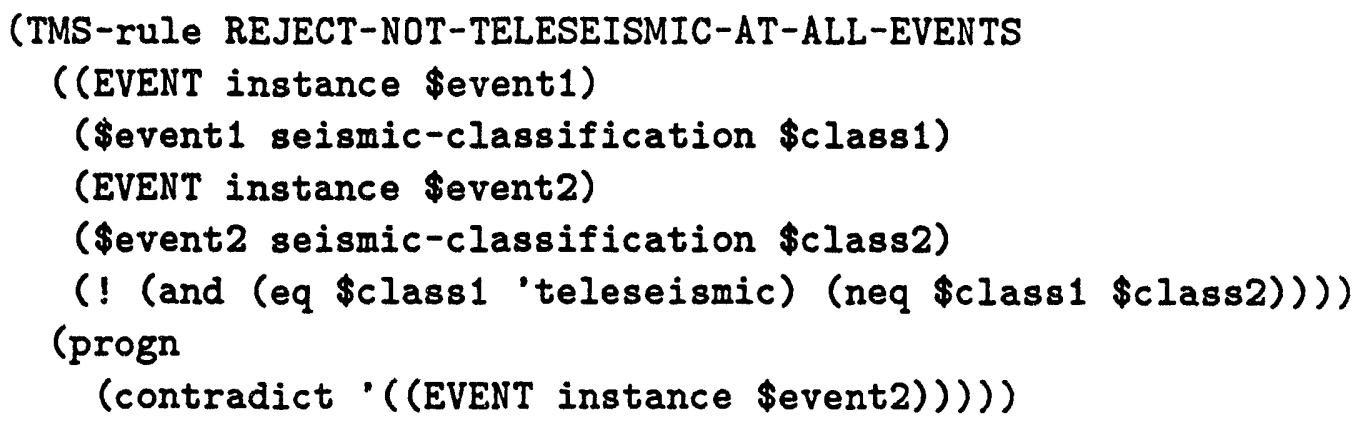

This truth maintenance rule maintains that it is known in a local regional network, a teleseismic event should be interpreted as teleseismic at all the stations. If it is not classified as teleseismic at all the stations, then the inferencing chain along a teleseismic belief must be incorrect, and the partial interpretation is contradicted.

\section{Seismic Interpretation Parameters}

In this study, the class of events for which we sought to obtain full interpretations were regional; i.e., events whose seismic activity can be detected about a $1000 \mathrm{~km}$ away.

An interpretation consists of identifying the class of an event and determining its epicenter. To demonstrate the feasibility and to determine the usefulness of fuzzy set theory in seismic event interpretation show just one example from each class. A complete performance evaluation will require us to analyze the results on a larger database of events which we plan to conduct in our next report. The rule base that we have developed runs the Kohonen classifier to obtain the fuzzy classifications. For the limited set of events in this study, we were only interested in developing detailed rules for regional events. 
The general strategy of the rule base for regional events is to run simple phase picking algorithms to obtain an initial set of unlabeled picks. An image understanding algorithm is then applied to the spectrogram of the waveform to filter "picks" outside the region of interest. Depending on the number of picks left and the relationships between the picks, rules are fired to attach labels to the picks. Depending on the labels, a single station event epicenter is determined. To determine whether events between stations are associated, we used the criteria that (i) the difference in origin times must be less than the uncertainty in origin times, and (ii) the distance circles from the stations to the event should intersect. The associated picks from the different stations are then combined to obtain a network event interpretation.

\section{Results and Discussion}

In this section, we discuss examples of interpretations made using the fuzzy neural network system running on a SUN workstation. The database of events is from the LLNL regional network, which is located in the southwestern United States and is operated by the LLNL Treaty Verification Program. The events recorded by this four-station network are mostly local and regional events.

Figure 3.1 is an interpretation of an event detected at the Kanab station. After making a series of signal measurements, including spectrogram estimation and SONN fuzzy classification, and then applying the fuzzy inference engine, the system interpretes the event as a moving vehicle, such as an automobile, with a confidence value of 1.0. The confidence value is the final compatability value along a particular line of reasoning. Note that since we are using a handful of regional rules, the regional picks shown in Figure 3.1 are finally rejected by the rules for this event. The automobile interpretation is based only on the fuzzy neural network.

An example of a local event is shown in Figure 3.2. The system determines that the event is a local event with a confidence level of 0.63 .

Detections resulting from noise are quite common. An useful interpretation system ought to be able to determine if the detection is due to some particular noise source. Figure 3.3 is an interpretation of a noise event recorded at the Kanab station; the interpretation confidence level is 0.57 .

Figure 3.4 is an example of a regional event with a confidence level of 0.71 , recorded at the Landers station. Finally, Figure 3.5 depicts a teleseismic event recorded also at the Landers station and given a confidence level of .71 . 
From the performance of the system on a limited number (43) of events, we conclude that the system is able to perform quite well the fuzzy event interpretation for the different classes of events. We hope to perform a systematic evaluation of the performance of a similar system with a larger number of events in the future. However, to make the system more portable and reliable, we need to develop many more rules and integrate this system with other image-understanding algorithms. In addition to developing rules, we can develop a suite of linguistic variables in our problem domain. Obvious linguistic variable candidates can be used to model the simple phase-picking algorithm and phase associations with fuzzy sets to improve the accuracy of interpretations within a single class.

We conclude by noting that the system developed is quite general and can used in many different types of problems where one needs to deal with uncertainty, ambiguity, and vagueness. Many nonprolieration monitoring problems fall into these categories and we hope that the methodology developed will be applied to other problems.

\section{Acknowledgments}

The authors would like to thank Jim Hannon and Lew Glenn of the LLNL Treaty Verification Program for encouraging us to explore the use of fuzzy logic system in seismic data interpretation. We thank Elaine Price for her editorial help in the writing of this report.

\section{References}

Bache, T. C., S. R. Bratt, J. Wang, R. M. Fung, C. Kobryn, and J. W. Given (1990). The intelligent monitoring system. Bull. Seis. Soc. Am. 80, 1833-1851.

Doyle, J. (1979). A Truth Maintenance System, Artificial Intelligence, 12

deKleer, J. (1984). Choices without backtracking, Proc. of the National Conference on Artificial Intelligence, Austin, Texas.

Dubois, Prade (1988). An Introduction to Possibilistic and Fuzzy Logics, Readings in Uncertain Reasoning, Morgan Kaufman pp. 742-761

Johnson, R. R., T. W. Canales, D. L. Lager, C. L. Mason, and R. M. Searfus. (1987). Interpreting Signals with an Assumption-Based Truth Maintenance System. Proc. SPIE, vol. 786, pp. 332-337.

Kosko, B. (1992). Neural Networks and Fuzzy Systems, Prentice Hall, pp. 299335 
Maurer, W. J., Dowla F. U., Jarpe S. P. (1992)., Seismic Event Interpretation using self-organizing neural network. Proc. SPIE, vol. 1709, pp. 950-958.

Zadeh, L. (1982). Test-score semantics for natural languages and meaning representation via PRUF., Empirical Semantics, Brockmeyer, Bochum, pp. 281-349. Zadeh, L. (1975). The Concept of a Linguistic Variable and its Application to Approximate Reasoning, Information Sciences, vol. 9, pp. 199-249. 


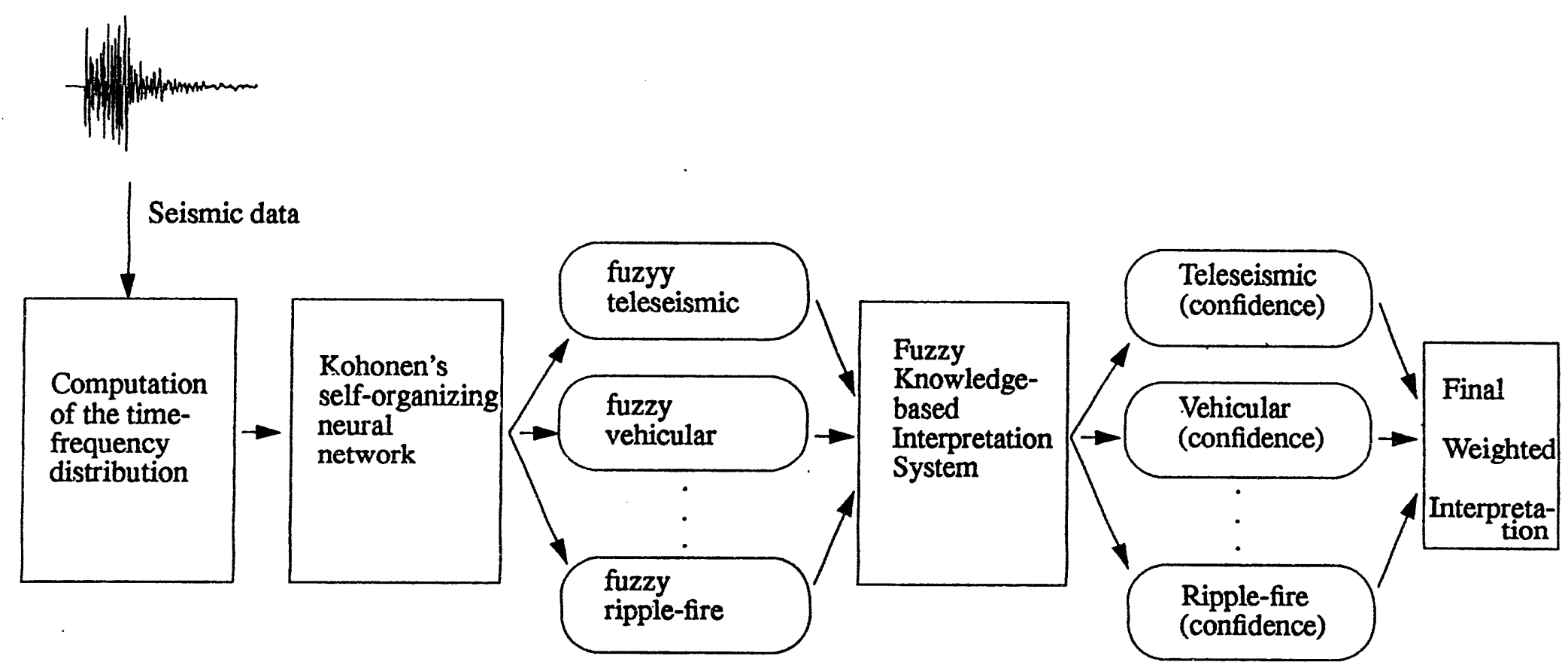

Figure 1: The fuzzy classification approach: a framework for automated interpretation of seismic events. The use of self-organizing neural network, fuzzy rules, and rule-based expert systems makes this approach useful for single station seismic event interpretation. 


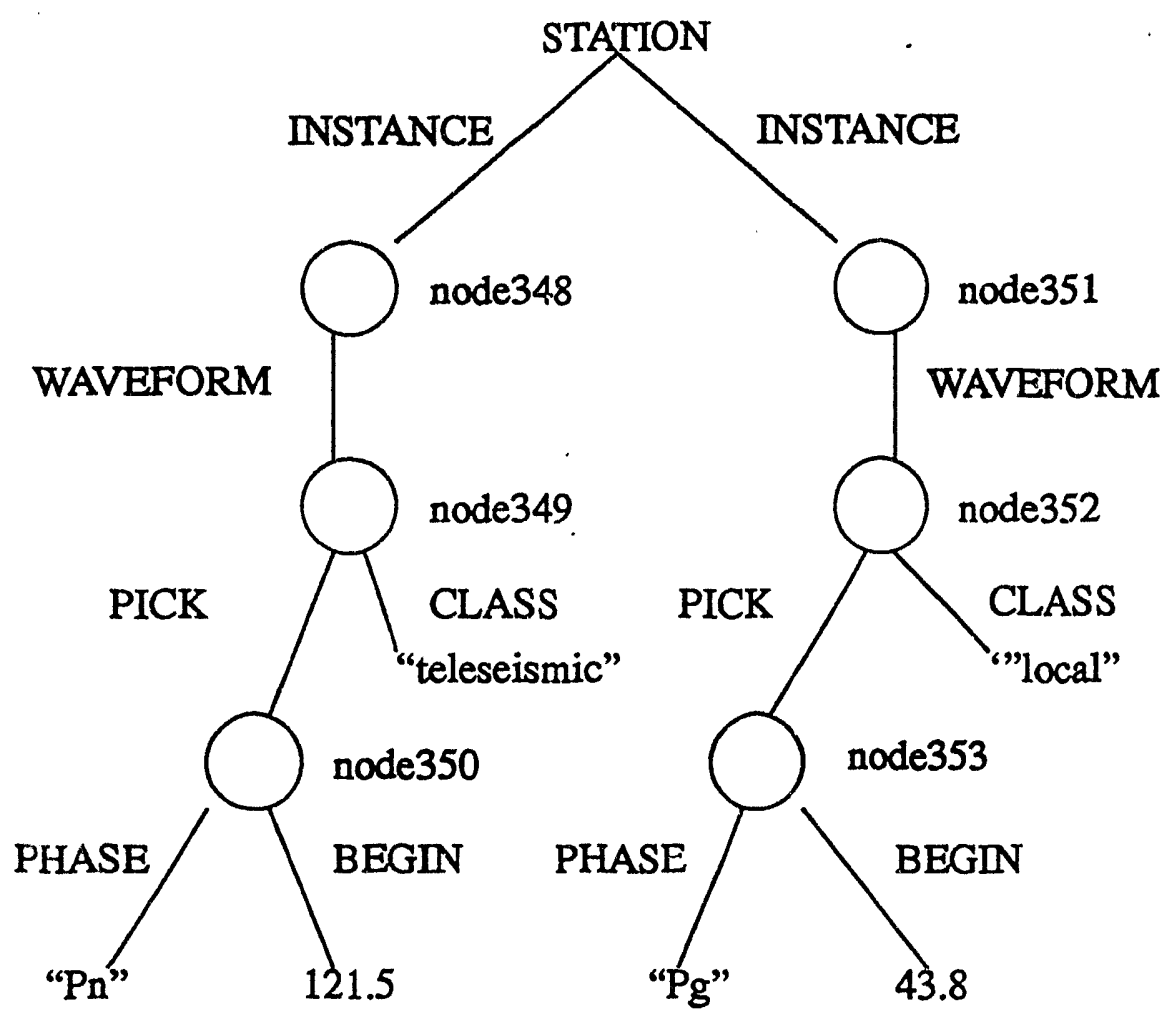

Figure 2. The semantic network formalism. Facts are represented as a <node,link,node> triple. Measurements from data are stored in the semantic network and opertated on by the fuzzy logic rules. 
Data:


System Interpretation:

\begin{tabular}{|l|c|c|c|c|c|c|}
\hline \multirow{2}{*}{ Event } & \multirow{2}{*}{ Station } & \multicolumn{5}{|c|}{ Interpretation } \\
\hline & & Auto Local & Noise & Regional Teleseismic \\
\hline 900882143 & Kanab & 1.0 & .44 & .35 & .28 & .11 \\
\hline
\end{tabular}

Figure 3.1: Automated fuzzy neural network interpretation of an event due to an automobile. 
Data:

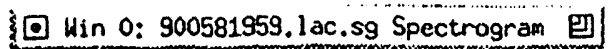

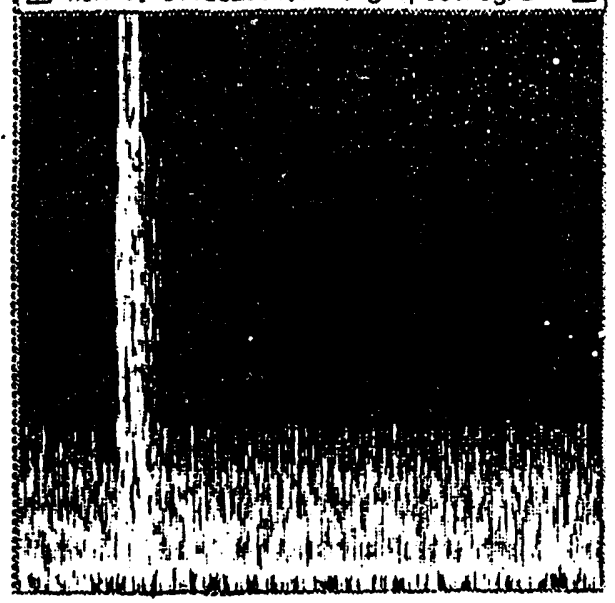

Measurements

1.achfz
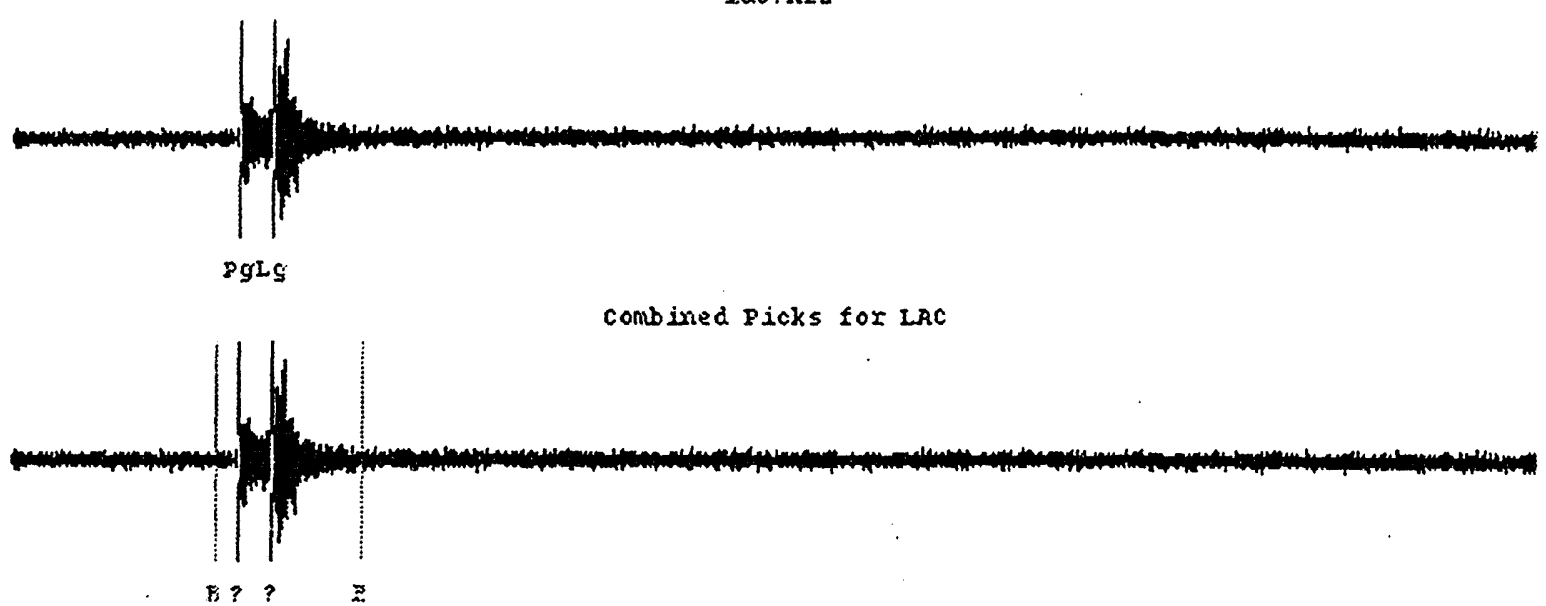

3c Picks fOr LAC

System Interpretation:

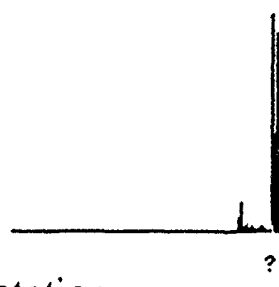

\begin{tabular}{|l|l|l|l|l|l|l|}
\hline \multirow{2}{*}{ Event } & \multirow{2}{*}{ Station } & \multicolumn{5}{|c|}{ Interpretation } \\
\hline 900581959 & Landers & .0 & .63 & .13 & .25 & .0 \\
\hline
\end{tabular}

Figure 3.2: Automated fuzzy neural network interpretation of an event due to a local event.. 
Data:


System Interpretation:

\begin{tabular}{|l|l|l|l|l|l|c|}
\hline Event & Station & \multicolumn{5}{|c|}{ Auto Local Noise } \\
\hline 900452352 & Kanab & .14 & .25 & .57 & .31 & .22 \\
\hline
\end{tabular}

Figure 3.3: Automated fuzzy neural network interpretation of an event due to a noise event. 
Data:
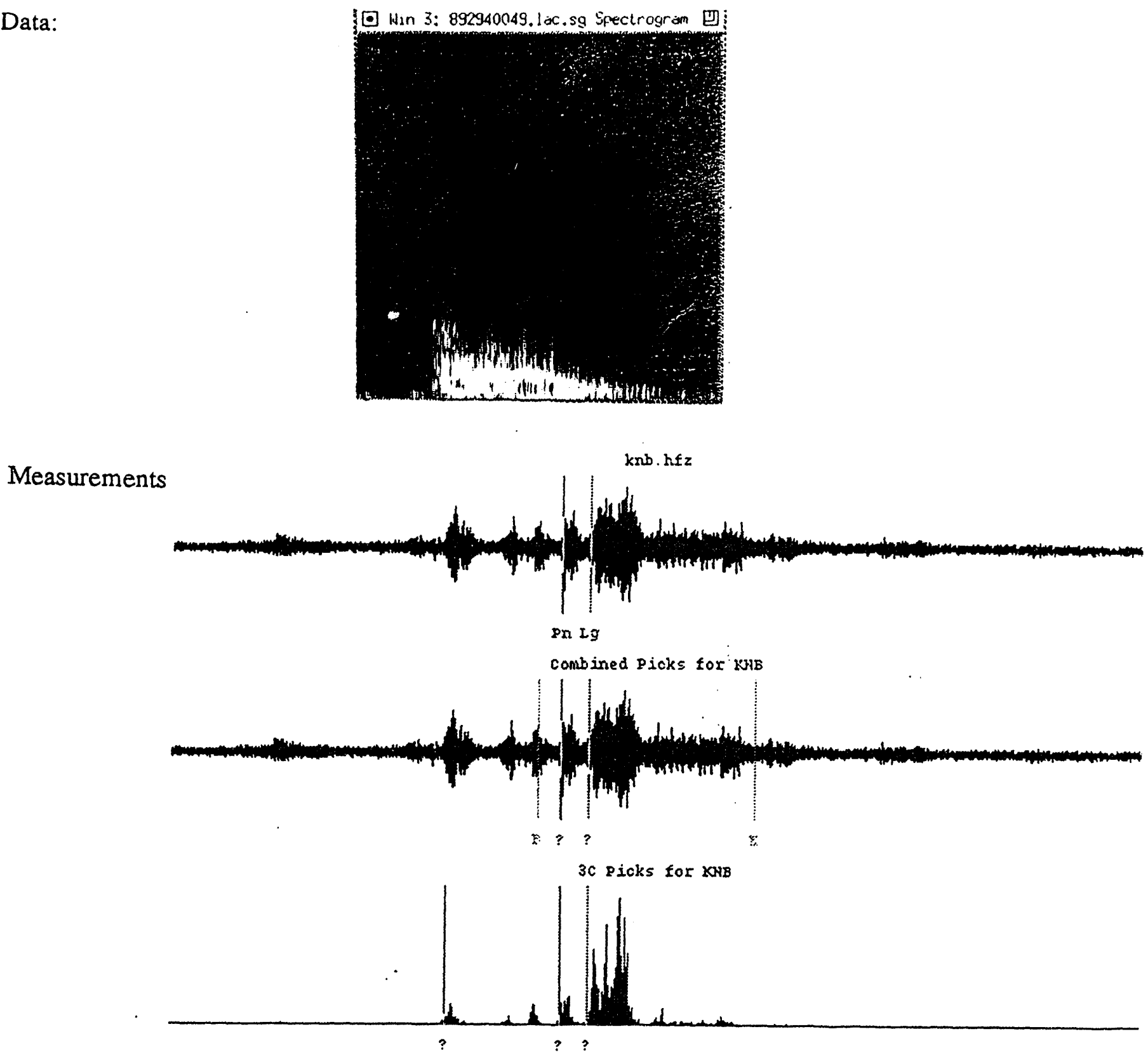

System Interpretation:

\begin{tabular}{|l|c|c|c|c|c|c|}
\hline Event & Station & \multicolumn{5}{|c|}{ Interpretation } \\
\hline 892940049 & Landers & .46 & .24 & .25 & .71 & .29 \\
\hline
\end{tabular}

Figure 3.4: Automated fuzzy neural network interpretation of an event due to a regional event. 
Data:

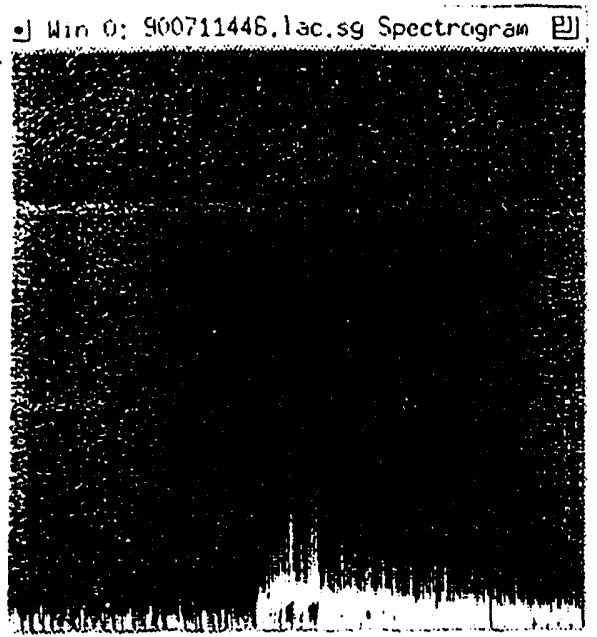

Measurements


$\$ ? ? ?$

3C Ficks for LRC

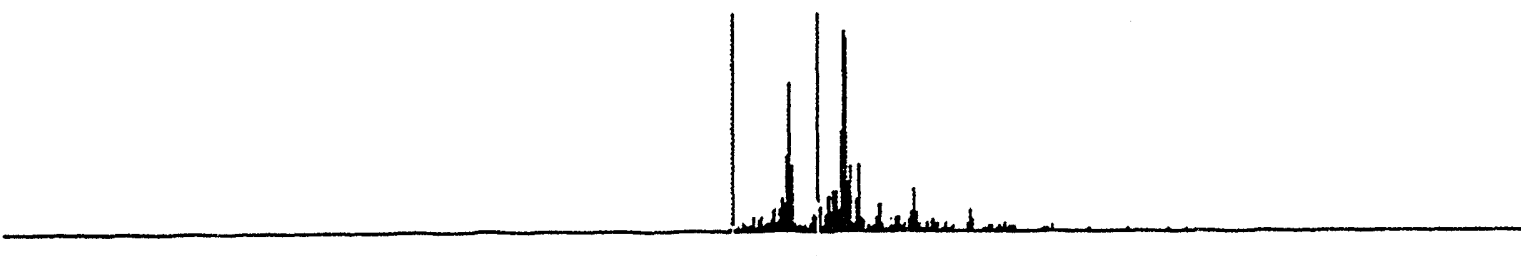

System Interpretation:

\begin{tabular}{|l|l|l|l|l|l|c|}
\hline \multirow{2}{*}{ Event } & Station & \multicolumn{5}{|c|}{ Interpretation } \\
\hline \multirow{2}{*}{900711446} & Landers & .34 & .33 & .36 & .29 & .71 \\
& & & & & & \\
\hline
\end{tabular}

Figure 3.5: Automated fuzzy neural network interpretation of an event due to a teleseismic event. 

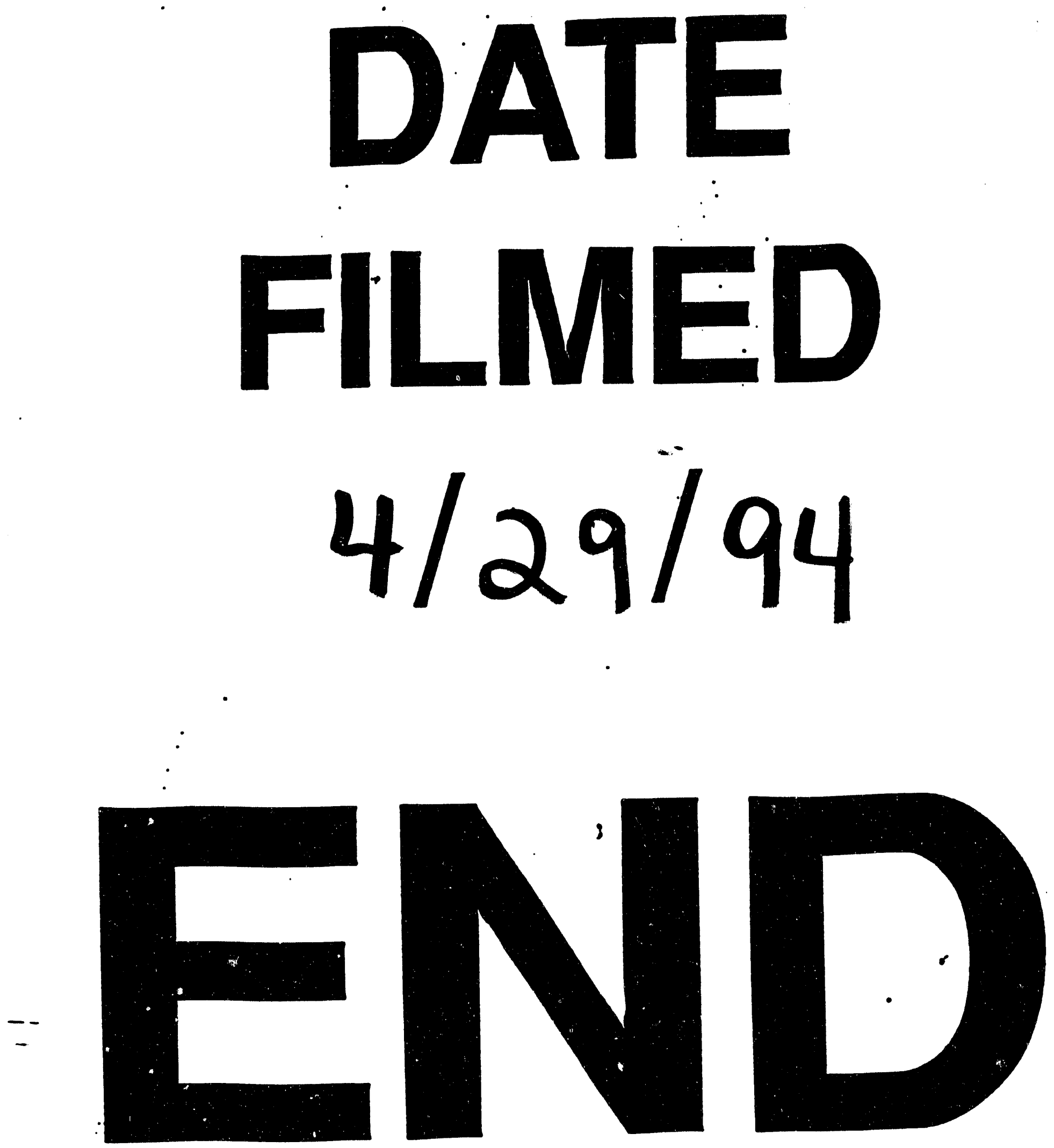
